\title{
Effect of Mg doping in the barriers on the electrical performance of InGaN/ GaN-based light-emitting diodes
}

\author{
Binbin Zhu ${ }^{\text {a,b }}$, Zi-Hui Zhang ${ }^{\text {a,c }}$, Swee Tiam Tan ${ }^{\text {a }}$, Shunpeng Lu ${ }^{\text {a }}$, Yiping Zhang ${ }^{\text {a }}$, Xuejun Kang ${ }^{\text {a }}$, \\ Ning Wang a , Namig Hasanov ${ }^{a}$, Hilmi Volkan Demir ${ }^{\text {a,b, d, }}$ \\ ${ }^{a}$ Luminous! Centre of Excellence for Semiconductor Lighting and Displays, The Photonics Institute, School of Electrical and Electronic Engineering, Nanyang Technological \\ University, 50 Nanyang Avenue, Singapore, 639798, Singapore \\ ${ }^{\mathrm{b}}$ School of Physics and Mathematical Sciences, Nanyang Technological University, 21 Nanyang Link, Singapore, 637371, Singapore \\ ${ }^{\mathrm{c}}$ Key Laboratory of Electronic Materials and Devices of Tianjin, School of Electronics and Information Engineering, Hebei University of Technology, 5340 Xiping Road, \\ Beichen District, Tianjin, 300401, PR China \\ ${ }^{\mathrm{d}}$ Department of Physics, UNAM-Institute of Material Science and Nanotechnology, Bilkent University, Ankara, TR-06800, Turkey
}

In this work, we report how the Mg doping in the barriers affects the electrical performance of InGaN/GaN-based light-emitting diodes. When compared with the reference device that does not have $\mathrm{Mg}$ doped quantum barriers, the turn-on voltage for the proposed device is reduced and the electrical thermal stability is improved. The superior electrical performance is analyzed through the temperature dependent currentvoltage and capacitance-voltage characteristics. Meanwhile a reduced depletion length and increased acceptor concentration are achieved in the control devices which is consistent with the simulated results.

During the last few decades, due to many advantages such as long lifetime, durable quality and high efficiency, InGaN/GaN-based lightemitting diodes (LEDs) have achieved widespread applications in many aspects, such as general lighting, LCD backlights and automobile lighting [1]. However, plenty of research in this field was focused on their optical properties in the last few years, and the investigation on electrical properties is not conducted carefully [2,3]. As one of the most important parameters, worse electrical property can directly leads more heat, which will induce the degradation of LED performance and the decreasing of the wall-plug efficiency simultaneously [4]. Besides, studies on the electrical properties can help to uncover the inherent physics of LED devices $[2,5]$. For example, the capacitance-voltage (C-V) characteristics can be used to reveal the depletion region widths at various biases [6]. Beside, tt has been reported that $\mathrm{Mg}$ doping in the quantum barriers is promising to improve the LED external quantum efficiency effectively [7]. However, the impact about how the Mg doping in the quantum barriers affects the electrical property is not fully studied yet.

In this work, we compare the electrical characteristics of InGaN/GaNbased LEDs for the sample with Mg doping in the barriers and the reference without $\mathrm{Mg}$ doping the quantum barriers. The mechanism for the reduced turn-on voltage and improved thermal stability is studied and analyzed through capacitance-voltage characteristics and selfconsistent simulation.

The LED epi-layers were grown on c-plane sapphire substrates by metal-organic chemical vapor deposition (MOCVD) system, with schematic diagrams as illustrated in Fig. 1. For LED A, the growth was initiated on a $30 \mathrm{~nm}$-thick $\mathrm{GaN}$ nucleation layer [8]. Then, a $2 \mu \mathrm{m}$-thick un-intentionally doped GaN was grown as the buffer layer, which was then followed by a $4 \mu \mathrm{m}$-thick n-doped GaN layer with a doping concentration of $5 \times 10^{18} \mathrm{~cm}^{-3}$. Four pairs of $\operatorname{In}_{0.15} \mathrm{Ga}_{0.85} \mathrm{~N} / \mathrm{GaN}$ quantum wells were grown then and the thicknesses of quantum barriers and quantum wells were $12 \mathrm{~nm}$ and $3 \mathrm{~nm}$, respectively. Finally, a p-type electron blocking layer (EBL) of $20 \mathrm{~nm}$-thick $\mathrm{Al}_{0.15} \mathrm{Ga}_{0.85} \mathrm{~N}$ was grown and it was then capped by a $200 \mathrm{~nm}$-thick p-type GaN with a doping concentration of $3 \times 10^{17} \mathrm{~cm}^{-3}$. After the growth, LED A was annealed at the temperature of $700{ }^{\circ} \mathrm{C}$ in $\mathrm{N}_{2}$ ambient to activate p-type dopants. LED B is the proposed sample with $\mathrm{Mg}$ doping in the quantum barriers, the growth of which was the same as LED A except that the middle $6 \mathrm{~nm}$ region in each quantum barriers was doped by $\mathrm{Mg}$ dopants with a doping concentration of $3 \times 10^{17} \mathrm{~cm}^{-3}$. The structure for selectively $\mathrm{Mg}$ doping in each quantum barrier was to suppress the $\mathrm{Mg}$ diffusion into quantum wells. After epitaxial growth, both LED A and LED B were fabricated into standard LED chips. $\mathrm{Ni}(5 \mathrm{~nm}) / \mathrm{Au}(5 \mathrm{~nm})$ layer was deposited and annealed firstly which acted as the current spreading layer. Then, the mesa area with the size of $1 \mathrm{~mm} \times 1 \mathrm{~mm}$ was obtained by inductively coupled plasma (ICP) etching, and $\mathrm{Ti} / \mathrm{Ag} / \mathrm{Ti} / \mathrm{Au}$ was deposited as electrodes for both $\mathrm{n}$ and $\mathrm{p}$ contacts. The current-voltage characteristic was measured by the SC-200-mm prober station, and the temperature was controlled by the hot plate with the values ranging from $25{ }^{\circ} \mathrm{C}$ to $150{ }^{\circ} \mathrm{C}$. The capacitance-voltage characteristic was also measured by the SC-200-mm prober station, with the AC signal voltage of $20 \mathrm{mV}$ and frequency of $1 \mathrm{MHz}$.

\footnotetext{
* Corresponding author. Luminous! Centre of Excellence for Semiconductor Lighting and Displays, The Photonics Institute, School of Electrical and Electronic Engineering, Nanyang Technological University, 50 Nanyang Avenue, Singapore, 639798, Singapore.

E-mail address: volkan@stanfordalumni.org (H.V. Demir).
} 


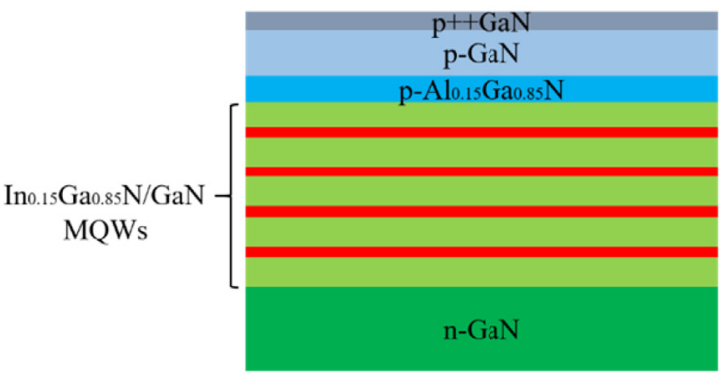

LED A

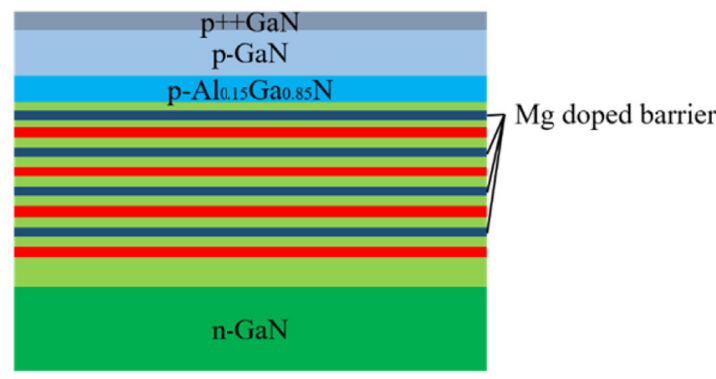

LED B

Fig. 1. Schematic diagrams for studied LED structures, in which LED A is the reference and LED B is the sample with Mg doping in the quantum barriers.

Furthermore, in order to reveal the physical insights, we also performed numerical simulations by APSYS software, which selfconsistently solves the Poisson equation, continuity equation and Schrödinger's equation with proper boundary conditions. In our simulation, the Auger recombination coefficient is taken to be $1 \times 10^{-30}$ $\mathrm{cm}^{6} \mathrm{~s}^{-1}$ [9] and the SRH lifetimes for both electron and hole are $43 \mathrm{~ns}$ $[9,10]$. The polarization charge is set as $40 \%$ of theoretical value due to crystal relaxation [11]. The other parameters can be found in reference [11].

Fig. 2 (a) shows the experimental $I-V$ characteristics for both devices measured under different temperatures. Here, we choose the current of $20 \mathrm{~mA}$ as the reference for electrical analysis. The forward voltage at room temperature for LED A is $3.37 \mathrm{~V}$ and for LED B is $2.95 \mathrm{~V}$, which means the voltage is decreased by $0.42 \mathrm{~V}$ for LED B. For the temperature dependent $I-V$ curve, temperatures are chosen as $25^{\circ} \mathrm{C}, 50^{\circ} \mathrm{C}, 75^{\circ} \mathrm{C}$, $100^{\circ} \mathrm{C}$, and $150^{\circ} \mathrm{C}$. The forward voltages for LED A at different temperatures are $3.37,3.26,3.15,3.04$ and $2.86 \mathrm{~V}$ respectively, while the values for LED B are $2.95,2.88,2.82,2.76$ and $2.63 \mathrm{~V}$ respectively. The decreasing values of the forward voltages are $0.11,0.11,0.11$ and $0.18 \mathrm{~V}$ with increasing temperature for LED A, while those of LED B are 0.07, $0.06,0.06$ and $0.13 \mathrm{~V}$ respectively. Thus, we can conclude the thermal stability of electrical property is better for LED B than that for LED A. The reduced forward-voltage and improved thermal stability for LED A will be explained as follows.

Fig. 3(a) shows the capacitance-voltage characteristics for both samples at room temperature. At zero bias voltage, the capacitance of the LEDs is mainly composed of the junction capacitance, which can be expressed as

$C=\varepsilon A / L$,
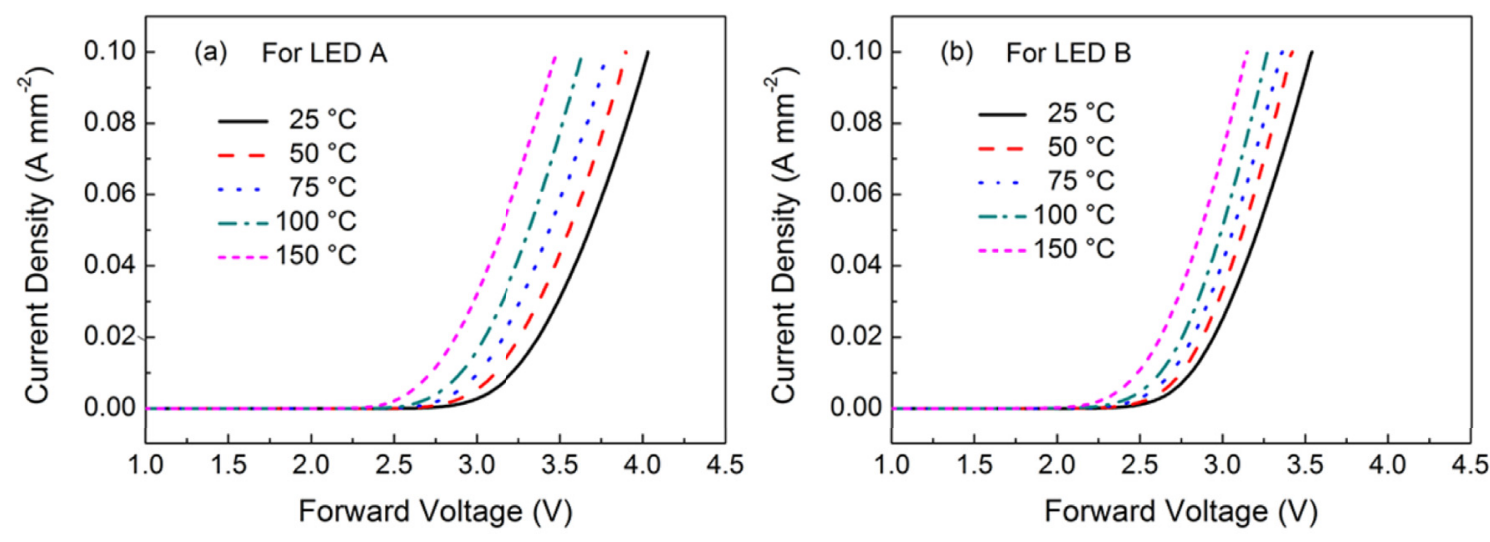

Fig. 2. $I-V$ characteristics under temperatures from $25^{\circ} \mathrm{C}$ to $150^{\circ} \mathrm{C}$ for (a) LED A and (b) LED B.
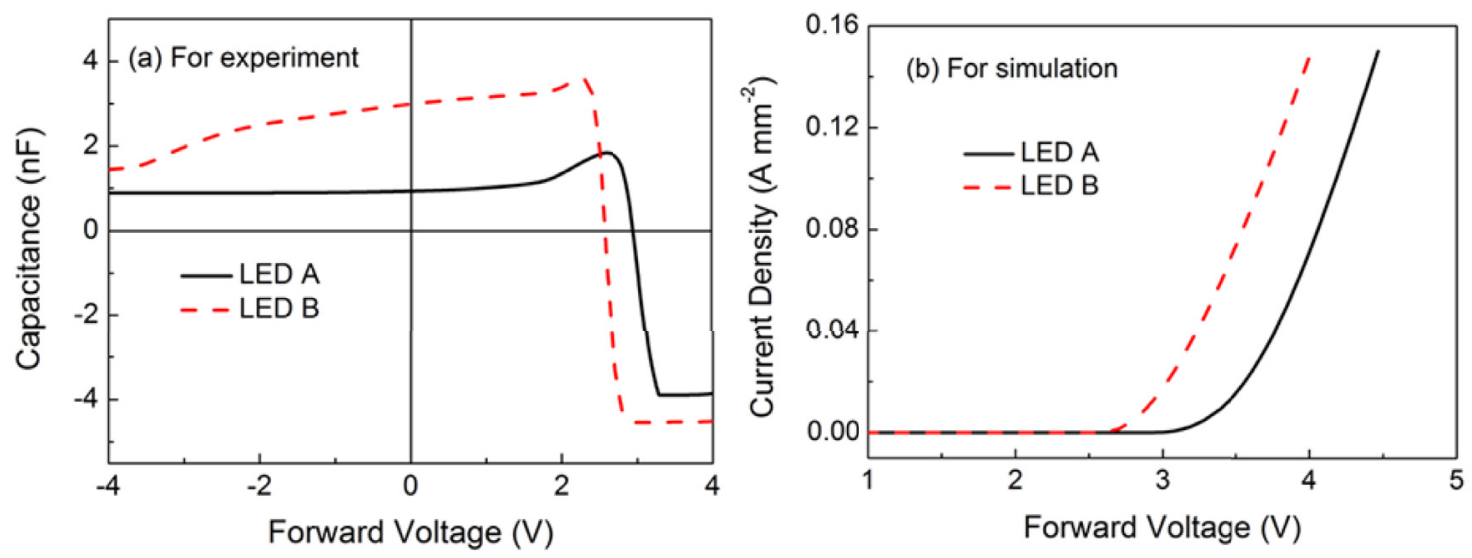

Fig. 3. (a) Capacitance-Voltage characteristics, and (b) simulated $I-V$ characteristics for both samples at room temperature. 

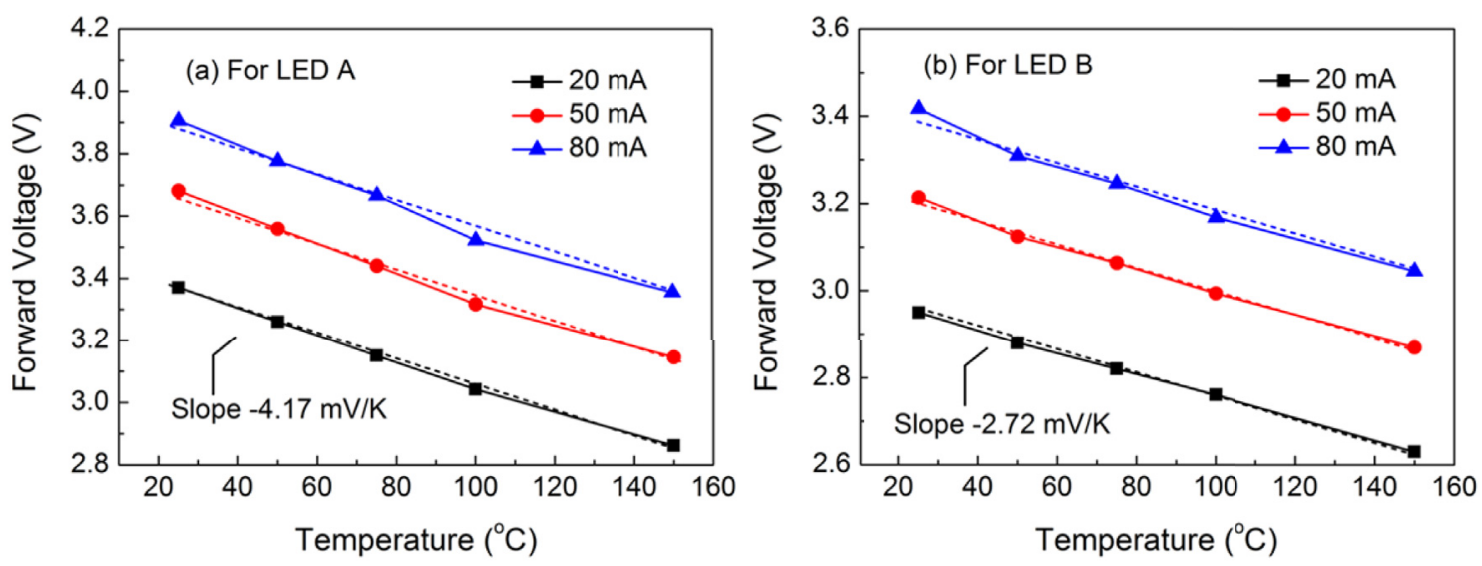

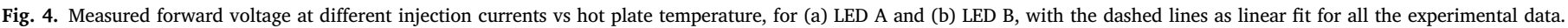

in which $\varepsilon$ represents the permittivity with the value of $8.5 \varepsilon_{0}, A$ is the area of the junction with the value of $1 \times 1 \mathrm{~mm}^{2}$, and $L$ is the length of the depletion region [6]. From the experimental data based on Fig. 3(a), the junction capacitance is $0.94 \mathrm{nF}$ for LED A, and $2.98 \mathrm{nF}$ for LED B. Then we can calculate the depletion length based on equation (a), which is $76.5 \mathrm{~nm}$ for LED A and $23.7 \mathrm{~nm}$ for LED B. In the simulation, the voltage was set at $0.1 \mathrm{~V}$ which could be compared with zero bias. The depletion length was extracted from the calculated capacitance at $1 \mathrm{MHz}$ and value is $76 \mathrm{~nm}$ for LED A and $33 \mathrm{~nm}$ for LED B. The trend for the change of the depletion length is consistent with the experimental results. From Fig. 3(a), we can also see that the capacitance starts to decrease when the voltage reaches a value and even become negative when the forward voltage is large enough. The decrease of capacitance is caused by the carriers' recombination in the quantum wells [12], and the voltages at peak capacitances for both samples are $2.65 \mathrm{~V}$ and $2.26 \mathrm{~V}$, respectively. The difference between the carriers' recombination onset voltages for the two samples is similar to the difference between the forward voltages at $20 \mathrm{~mA}$, which is a direct evidence for the reduced forward voltage. Since the doping concentration in $\mathrm{n}-\mathrm{GaN}$ is much higher than that in p-GaN, the LED can be treated as a model of donor and acceptor doping with concentration $N_{D}$ on the $\mathrm{n}^{+}$-side and $N_{A}$ on the p-side, respectively. The acceptor concentration, $N_{A}$, can be calculated by the following equation [13],

$N_{A}=-\frac{2}{\varepsilon e A^{2}}\left[d\left(\frac{1}{C^{2}}\right) / d V\right]^{-1}$, if $N_{D} \gg N_{A}$,

from which, the acceptor doping concentration is $1.75 \times 10^{22} \mathrm{~m}^{-3}$ for LED A, and $1.22 \times 10^{23} \mathrm{~m}^{-3}$ for LED B. When $N_{D} \gg N_{A}$, the equation for the diffusion voltage can be expressed as equation (3) [14],

$V_{D}=\frac{W_{D}^{2}}{2 \varepsilon} N_{A}$,

in which $V_{D}$ is the diffusion voltage, $W_{D}$ is the depletion length and $N_{A}$ is the acceptor concentration. Both of the depletion length and the acceptor concentration can be calculated from the $C$ - $V$ curve. After we substitute both values into equation (3), the following relation can be achieved, while the subscript 1 and 2 denote LED A and LED B.

$V_{D 1}>V_{D 2}$,

Fig. 3(b) shows the simulated $I-V$ characteristics for both samples at room temperature, from which we can see that the forward voltage is also smaller for the proposed $\mathrm{Mg}$ doped sample, just as the shown experimental results.

Fig. 4 depicts the forward voltage vs temperature under different injection currents. The dashed lines are linear fit for all the experimental results. According to reference [15], a linear relationship between the junction temperature and forward voltage is found, while

$\frac{d V_{f}}{d T} \approx \frac{k}{T} \ln \left(\frac{N_{D} N_{A}}{N_{C} N_{V}}\right)-\frac{\alpha T(T+2 \beta)}{e(T+\beta)^{2}}-\frac{3 k}{e}$,

in which $\mathrm{k}$ is the Boltzmann constant, $\mathrm{T}$ is the temperature, $\alpha, \beta$ are constants, $N_{C}, N_{v}$ are effective density of states, and $N_{D}, N_{A}$ are donor and acceptor concentrations. $N_{C}, N_{v}$ are determined by the temperature [16]. Equation (5) is a lower limit for the magnitude of $d V_{f} / d T$, because the junction voltage is less than the built-in voltage in all practical cases [16]. According to the donor-acceptor model mentioned previously, they have the same donor concentration and the acceptor concentration is larger in the Mg doped sample. The value of $d V_{f} / d T$ should be larger in LED B. From Fig. 4 (a) and Fig. 4 (b), the temperature coefficients of the forward voltages at low current are $-4.17 \mathrm{mV} / \mathrm{K}$ and $-2.72 \mathrm{mV} / \mathrm{K}$, which is consistent with the analysis.

Besides the electrical property under normal operation, the current density before turn-on voltage is also measured. As shown in Fig. 5, the current density is larger in the sample with $\mathrm{Mg}$ doped barriers, which means the carriers is easier to pass the junction. However, this property may cause more current leakage and its property is slightly deteriorated.

In conclusion, InGaN/GaN-based LEDs with $\mathrm{Mg}$ doped barriers have been prepared and the improved electrical performance was realized and investigated. Temperature dependent $I-V$ characteristics and $C-V$

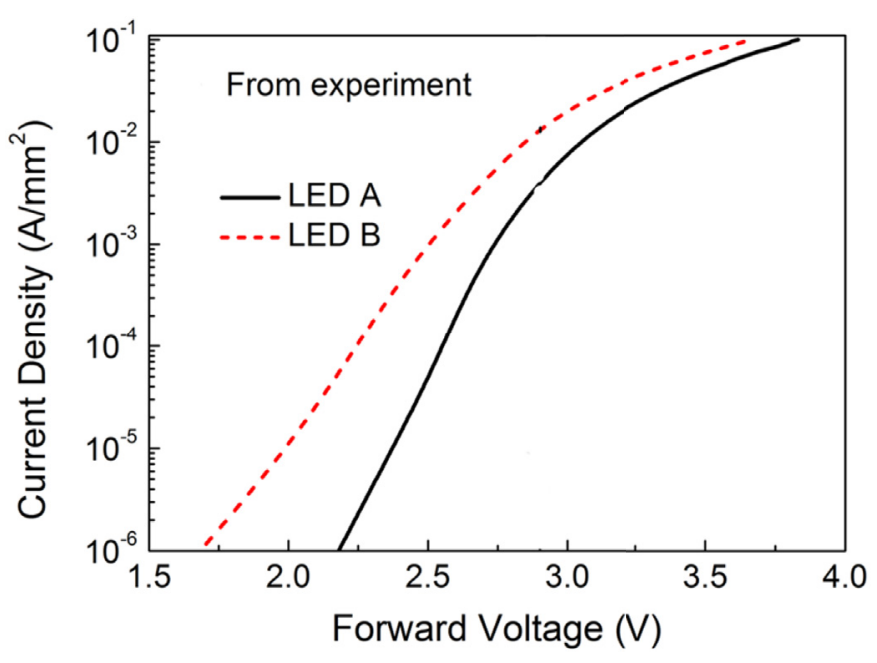

Fig. 5. Measured Current Density at low Forward Voltage. 
characteristic were analyzed and decreased depletion length was observed in the proposed sample. A donor-acceptor model was proposed and increased acceptor concentration was achieved. Based on the above analysis, our results offer meaningful physical insights on the mechanism how the Mg doped barrier affects the electrical performance of InGaN/ GaN-based LEDs.

This work was supported by the National Research Foundation of Singapore under Grant No. NRF-CRP-6-2010-2.

\section{Appendix A. Supplementary data}

Supplementary data related to this article can be found at https://doi. org/10.1016/j.physe.2017.12.025.

\section{References}

[1] S.T. Tan, X. Sun, H.V. Demir, S. DenBaars, Advances in the LED materials and architectures for energy-saving solid-state lighting toward "lighting revolution", Photonics J. IEEE 4 (2012) 613-619.

[2] L. Feng, D. Li, C. Zhu, C. Wang, H. Cong, G. Zhang, W. Du, Deep saturation of junction voltage at large forward current of light-emitting diodes, J. Appl. Phys. 102 (2007), 094511.

[3] Z.-H. Zhang, S.T. Tan, W. Liu, Z. Ju, K. Zheng, Z. Kyaw, Y. Ji, N. Hasanov, X.W. Sun, H.V. Demir, Improved InGaN/GaN light-emitting diodes with a p-GaN/n-GaN/pGaN/n-GaN/p-GaN current-spreading layer, Optics Exp. 21 (2013) 4958-4969.

[4] M. Krames, O. Shchekin, R. Mueller Mach, G. Mueller, L. Zhou, G. Harbers, M.G. Craford, Status and future of high-power light-emitting diodes for solid-state lighting, J. Disp. Technol. 3 (2007) 160-175.
[5] Y. Li, C. Wang, L. Feng, C. Zhu, H. Cong, D. Li, G. Zhang, Elucidating negative capacitance in light-emitting diodes using an advanced semiconductor device theory, J. Appl. Phys. 109 (2011), 124506.

[6] N. Chen, W. Lien, Y. Wang, H. Liu, Capacitance-voltage and current-voltage measurements of nitride light-emitting diodes, Electron Dev. IEEE Trans. 54 (2007) 3223-3228.

[7] Y. Ji, Z.-H. Zhang, S.T. Tan, Z.G. Ju, Z. Kyaw, N. Hasanov, W. Liu, X.W. Sun, H.V. Demir, Enhanced hole transport in InGaN/GaN multiple quantum well lightemitting diodes with a p-type doped quantum barrier, Optic Lett. 38 (2013) 202-204.

[8] Z.-H. Zhang, S. Tiam Tan, Z. Kyaw, Y. Ji, W. Liu, Z. Ju, N. Hasanov, X. Wei Sun, H. Volkan Demir, InGaN/GaN light-emitting diode with a polarization tunnel junction, Appl. Phys. Lett. 102 (2013), 193508-193508-193505.

[9] M. Meneghini, N. Trivellin, G. Meneghesso, E. Zanoni, U. Zehnder, B. Hahn, A combined electro-optical method for the determination of the recombination parameters in InGaN-based light-emitting diodes, J. Appl. Phys. 106 (2009), 114508.

[10] V. Fiorentini, F. Bernardini, O. Ambacher, Evidence for nonlinear macroscopic polarization in III-V nitride alloy heterostructures, Appl. Phys. Lett. 80 (2002) 1204-1206.

[11] I. Vurgaftman, J. Meyer, Band parameters for nitrogen-containing semiconductors, J. Appl. Phys. 94 (2003) 3675-3696.

[12] C. Zhu, L. Feng, C. Wang, H. Cong, G. Zhang, Z. Yang, Z. Chen, Negative capacitance in light-emitting devices, Solid State Electron. 53 (2009) 324-328.

[13] Y. Xia, E. Williams, Y. Park, I. Yilmaz, J. Shah, E. Schubert, C. Wetzel, Discrete steps in the capacitance-voltage characteristics of GaInN/GaN light emitting diode structures, in: MRS Proceedings, Cambridge Univ Press, 2004. E3. 38.

[14] E.F. Schubert, Light-emitting Diodes, Cambridge University Press, 2006.

[15] Y. Xi, E. Schubert, Junction-temperature measurement in GaN ultraviolet lightemitting diodes using diode forward voltage method, Appl. Phys. Lett. 85 (2004) 2163-2165.

[16] Y. Xi, T. Gessmann, J. Xi, J.K. Kim, J.M. Shah, E.F. Schubert, A.J. Fischer, M.H. Crawford, K.H. Bogart, A.A. Allerman, Junction temperature in ultraviolet light-emitting diodes, Jpn. J. Appl. Phys. 44 (2005) 7260. 